\title{
Learning Sequence Encoders for Temporal Knowledge Graph Completion
}

\author{
Alberto García-Durán ${ }^{1} \quad$ Sebastijan Dumančić $^{* 2} \quad$ Mathias Niepert $^{1}$ \\ ${ }^{1}$ NEC Labs Europe, Germany \\ ${ }^{2} \mathrm{KU}$ Leuven, Belgium \\ \{alberto.duran, mathias.niepert\}@neclab.eu \\ sebastijan. dumancic@cs.kuleuven.be
}

\begin{abstract}
Research on link prediction in knowledge graphs has mainly focused on static multirelational data. In this work we consider temporal knowledge graphs where relations between entities may only hold for a time interval or a specific point in time. In line with previous work on static knowledge graphs, we propose to address this problem by learning latent entity and relation type representations. To incorporate temporal information, we utilize recurrent neural networks to learn timeaware representations of relation types which can be used in conjunction with existing latent factorization methods. The proposed approach is shown to be robust to common challenges in real-world KGs: the sparsity and heterogeneity of temporal expressions. Experiments show the benefits of our approach on four temporal KGs. The data sets are available under a permissive BSD-3 license ${ }^{1}$.
\end{abstract}

\section{Introduction}

Knowledge graphs (KGs) are used to organize, manage, and retrieve structured information. The incompleteness of most real-world KGs has stimulated research on predicting missing relations between entities. A KG is of the form $\mathcal{G}=(\mathcal{E}, \mathcal{R})$, where $\mathcal{E}$ is a set of entities and, $\mathcal{R}$ is a set of relation types or predicates. One can represent $\mathcal{G}$ as a set of triples of the form (subject, predicate, object), denoted as $(\mathrm{s}, \mathrm{p}, \mathrm{o})$. The link prediction problem seeks the most probable completion of a triple $(\mathrm{s}, \mathrm{p}, ?)$ or $(?, \mathrm{p}, \mathrm{o})$ (Nickel et al., 2016). We focus on temporal KGs where some triples are augmented with time information and the link prediction problem asks for the most probable completion given time information. More formally, a temporal $\mathrm{KG} \mathcal{G}=(\mathcal{E}, \mathcal{R}, \mathcal{T})$ is a $\mathrm{KG}$ where

\footnotetext{
${ }^{*}$ Work done while interning at NEC Labs Europe

${ }^{1}$ https://github.com/nle-ml/mmkb
}

facts can also have the form (subject, predicate, object, timestamp) or (subject, predicate, object, time predicate, timestamp), in addition to ( $\mathrm{s}, \mathrm{p}, \mathrm{o})$ triples. For instance, facts such as (Barack Obama, born, US, 1961) or (Barack Obama, president, US, occursSince, 2009-01) express temporal information about the facts associated with Barack Obama. While the former expresses that a relation type occurred at a specific point in time, the latter expresses an (open) time interval using the time predicate "occursSince." The latter example also illustrates a common challenge posed by the heterogeneity of time expressions due to variations in language and serialization standards.

Most approaches to link prediction are characterized by a scoring function that operates on the entity and relation type embeddings of a triple (Bordes et al., 2013; Yang et al., 2014; Guu et al., 2015). Learning representations that carry temporal information is challenging due to the sparsity and irregularities of time expressions. It is possible, however, to turn time expressions into sequences of tokens expressing said temporal information. Moreover, character-level architectures for language modeling (Zhang et al., 2015; Kim et al., 2016) operate on characters as atomic units to derive word embeddings. Inspired by these models, we propose a method to incorporate time information into standard embedding approaches for link prediction. We learn time-aware representations by training a recursive neural network with sequences of tokens representing the time predicate and the digits of the timestamp, if they exist. The last hidden state of the recurrent network is combined with standard scoring functions from the KG completion literature. 


\section{Related Work}

Reasoning with temporal information in knowledge bases has a long history and has resulted in numerous temporal logics (van Benthem, 1995). Several recent approaches extend statistical relational learning frameworks with temporal reasoning capabilities (Chekol et al., 2017; Chekol and Stuckenschmidt, 2018; Dylla et al., 2013).

There is also prior work on incorporating temporal information in knowledge graph completion methods. Jiang et al. (2016) capture the temporal ordering that exists between some relation types as well as additional common-sense constraints to generate more accurate link predictions. Esteban et al. (2016) introduce a prediction model for link prediction that assumes that changes to a $\mathrm{KG}$ are introduced by incoming events. These events are modeled as a separate event graph and used to predict the existence of links in the future. Trivedi et al. (2017) model the occurrence of a fact as a point process whose intensity function is influenced by the score assigned to the fact by an embedding function. Leblay and Chekol (2018) develop scoring functions that incorporate time representations into a TransE-type scoring function. Prior work has also incorporated numerical but non-temporal entity information for knowledge base completion (Garcia-Duran and Niepert, 2017).

Contrary to all previous approaches, we encode sequences of temporal tokens with an RNN. This facilitates the encoding of relation types with temporal tokens such as "since," "until," and the digits of timestamps. Moreover, the RNN encoding provides an inductive bias for parameter sharing among similar timestamps (e.g., those occurring in the same century). Finally, our method can be combined with all existing scoring functions.

\section{Time-Aware Representations}

Embedding approaches for $\mathrm{KG}$ completion learn a scoring function $f$ that operates on the embeddings of the subject $\mathbf{e}_{s}$, the object $\mathbf{e}_{o}$, and the predicate $\mathbf{e}_{p}$ of the triples. The value of this scoring function on a triple $(s, p, o), f(s, p, o)$, is learned to be proportional to the likelihood of the triples being true. Popular examples of scoring functions are

- TransE (Bordes et al., 2013)

$$
f(s, p, o)=\left\|\mathbf{e}_{s}+\mathbf{e}_{p}-\mathbf{e}_{o}\right\|_{2} .
$$

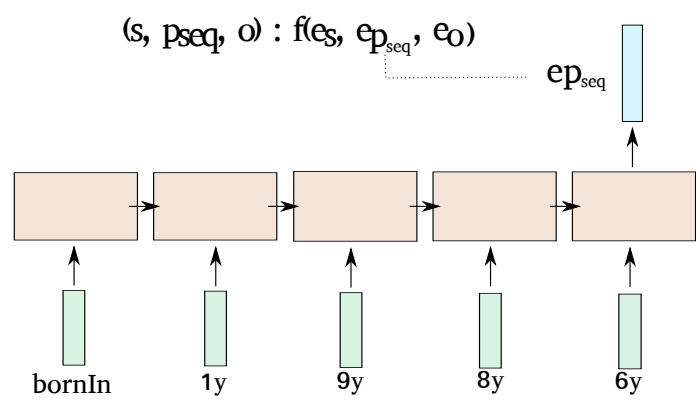

Figure 1: Learning time-aware representations.

- DistMult (Yang et al., 2014):

$$
f(s, p, o)=\left(\mathbf{e}_{s} \circ \mathbf{e}_{o}\right) \mathbf{e}_{p}^{T},
$$

where $\mathbf{e}_{s}, \mathbf{e}_{o} \in \mathbb{R}^{d}$ are the embeddings of the $s u b$ ject and object entities, $\mathbf{e}_{p} \in \mathbb{R}^{d}$ is the embedding of the relation type predicate, and $\circ$ is the elementwise product. These scoring functions do not take temporal information into account.

Given a temporal KG where some triples are augmented with temporal information, we can decompose a given (possibly incomplete) timestamp into a sequence consisting of some of the following temporal tokens

$$
\begin{aligned}
& \overbrace{0 \cdot 1 \cdot 2 \cdot 3 \cdot 4 \cdot 5 \cdot 6 \cdot 7 \cdot 8 \cdot 9}^{\text {year }} \\
& \overbrace{01 \cdot 02 \cdot 03 \cdot 04 \cdot 05 \cdot 06 \cdot 07 \cdot 08 \cdot 09 \cdot 10 \cdot 11 \cdot 12}^{\text {day }} \\
& \overbrace{0 \cdot 1 \cdot 2 \cdot 3 \cdot 4 \cdot 5 \cdot 6 \cdot 7 \cdot 8 \cdot 9}
\end{aligned}
$$

Hence, temporal tokens have a vocabulary size of 32. Moreover, for each triple we can extract a sequence of predicate tokens that always consists of the relation type token and, if available, a temporal modifier token such as "since" or "until." We refer to the concatenation of the predicate token sequence and (if available) the sequence of temporal tokens as the predicate sequence $p_{s e q}$. Now, a temporal $\mathrm{KG}$ can be represented as a collection of triples of the form $\left(s, p_{s e q}, o\right)$, wherein the predicate sequence may include temporal information. Table 1 lists some examples of such facts from a temporal KG and their corresponding predicate sequence. We use the suffix $y, m$ and $d$ to indicate whether the digit corresponds to year, month or day information. It is these sequences of tokens that are used as input to a recurrent neural network.

\subsection{LSTMs for Time-Encoding Sequences}

A long short-term memory (LSTM) is a neural network architecture particularly suited for modeling sequential data. The equations defining an 


\begin{tabular}{l|l}
\multicolumn{1}{c|}{ Fact } & \multicolumn{1}{c}{ Predicate Sequence } \\
\hline (Barack Obama, country, US) & [country] \\
(Barack Obama, born, US, 1961) & [born, 1y, 9y, 6y, 1y] \\
(Barack Obama, president, US, since, 2009-01) & [president, since, 2y, 0y, 0y, 9y, 01m]
\end{tabular}

Table 1: Facts and their corresponding predicate sequence.

\section{LSTM are}

$$
\begin{aligned}
& \mathbf{i}=\sigma_{g}\left(\mathbf{h}_{n-1} \mathbf{U}_{i}+\mathbf{x}_{n} \mathbf{W}_{i}\right) \\
& \mathbf{f}=\sigma_{g}\left(\mathbf{h}_{n-1} \mathbf{U}_{f}+\mathbf{x}_{n} \mathbf{W}_{f}\right) \\
& \mathbf{o}=\sigma_{g}\left(\mathbf{h}_{n-1} \mathbf{U}_{o}+\mathbf{x}_{n} \mathbf{W}_{o}\right) \\
& \mathbf{g}=\sigma_{c}\left(\mathbf{h}_{n-1} \mathbf{U}_{g}+\mathbf{x}_{n} \mathbf{W}_{g}\right) \\
& \mathbf{c}_{n}=\mathbf{f} \circ \mathbf{c}_{n-1}+\mathbf{i} \circ \mathbf{g} \\
& \mathbf{h}_{n}=\mathbf{o} \circ \sigma_{h}\left(\mathbf{c}_{n}\right)
\end{aligned}
$$

where $\mathbf{i}, \mathbf{f}, \mathbf{o}$ and $\mathbf{g}$ are the input, forget, output and input modulation gates, respectively. $\mathbf{c}$ and $\mathbf{h}$ are the cell and hidden state, respectively. All vectors are in $\mathbb{R}^{h} . \mathbf{x}_{n} \in \mathbb{R}^{d}$ is the representation of the n-th element of a sequence. In this paper we set $h=d . \sigma_{g}, \sigma_{c}$ and $\sigma_{h}$ are activation functions.

Each token of the input sequence $p_{\text {seq }}$ is first mapped to its corresponding $d$-dimensional embedding via a linear layer and the resulting sequence of embeddings used as input to the LSTM. Each predicate sequence of length $N$ is represented by the last hidden state of the LSTM, that is, $\mathbf{e}_{p_{\text {seq }}}=\mathbf{h}_{N}$. The predicate sequence representation, which carries time information, can now be used in conjunction with subject and object embeddings in standard scoring functions. For instance, temporal-aware versions of TRANSE and DistMult, which we refer to as TA-TRANSE and TA-DISTMULT, have the following scoring function for triples $\left(s, p_{s e q}, o\right)$ :

TA-TransE: $f\left(s, p_{s e q}, o\right)=\left\|\mathbf{e}_{s}+\mathbf{e}_{p_{s e q}}-\mathbf{e}_{o}\right\|_{2}$ TA-DistMult: $f\left(s, p_{s e q}, o\right)=\left(\mathbf{e}_{s} \circ \mathbf{e}_{o}\right) \mathbf{e}_{p_{s e q}}^{T}$.

All parameters of the scoring functions are learned jointly with the parameters of the LSTMs using stochastic gradient descent.

The advantages of character level models to encode time information for link prediction are: (1) the usage of digits and modifiers such as "since" as atomic tokens facilitates the transfer of information across similar timestamps, leading to higher efficiency (e.g. small vocabulary size); (2) at test time, one can obtain a representation for a timestamp even though it is not part of the training set;
(3) the model can use triples with and without temporal information as training data. Figure 1 illustrates the generic working of our approach.

\section{Experiments}

We conducted experiments on four different $\mathrm{KG}$ completion data sets where a subset of the facts are augmented with time information.

\subsection{Datasets}

Integrated Crisis Early Warning System (ICEWS) is a repository that contains political events with a specific timestamp. These political events relate entities (e.g. countries, presidents...) to a number of other entities via logical predicates (e.g. 'Make a visit' or 'Express intent to meet or negotiate'). Additional information can be found at http://www.icews.com/. The repository is organized in dumps that contain the events that occurred each year from 1995 to 2015 . We created two temporal KGs out of this repository, i) a short-range version that contains all events in 2014, and ii) a long-range version that contains all events occurring between 2005-2015. We refer to these two data sets as ICEWS 2014 and ICEWS 2005-15, respectively. Due to the large number of entities we selected a subset of the most frequently occurring entities in the graph and all facts where both the subject and object are part of this subset of entities. We split the facts into training, validation and test in a proportion of $80 \% / 10 \% / 10 \%$, respectively. The protocol for the creation of these data sets is identical to the onw followed in previous work (Bordes et al., 2013). To create YAGO15K, we used FREEBASE15K (Bordes et al., 2013) (FB15K) as a blueprint. We aligned entities from FB15K to YAGO (Hoffart et al., 2013) with SAMEAs relations contained in a YAGO dump ${ }^{2}$, and kept all facts involving those entities. Finally, we augment this collection of facts with time information from the "yagoDateFacts"3 dump. Contrary to the

\footnotetext{
2/yago-naga/yago3.1/yagoDBpediaInstances.ttl.7z

3/yago-naga/yago3.1/yagoDateFacts.ttl.7z
} 


\begin{tabular}{|l|c|c|c|c|}
\hline Data set & YAGO15K & ICEWS '14 & ICEWS 05-15 & WIKIDATA \\
\hline Entities & 15,403 & 6,869 & 10,094 & 11,134 \\
Relationships & 34 & 230 & 251 & 95 \\
\#Facts & 138,056 & 96,730 & 461,329 & 150,079 \\
\#Distinct TS & 198 & 365 & 4,017 & 328 \\
Time Span & $1513-2017$ & 2014 & $2005-2015$ & $25-2020$ \\
\hline \multirow{2}{*}{ Training } & 110,441 & 72,826 & 368,962 & 121,422 \\
& {$[29,381]$} & {$[72,826]$} & {$[368,962]$} & {$[121,422]$} \\
Validation & 13,815 & 8,941 & 46,275 & 14,374 \\
& {$[3,635]$} & {$[8,941]$} & {$[46,275]$} & {$[14,374]$} \\
Test & 13,800 & 8,963 & 46,092 & 14,283 \\
& {$[3,685]$} & {$[8,963]$} & {$[46,092]$} & {$[14,283]$} \\
\hline
\end{tabular}

Table 2: Statistics of the data sets. TS stands for timestamps. The number of facts with time information is in brackets.

\begin{tabular}{|l|cccc|cccc|}
\cline { 2 - 9 } \multicolumn{1}{c|}{} & \multicolumn{4}{c|}{ YAGO15K } & \multicolumn{4}{c|}{ WIKIDATA } \\
\cline { 2 - 9 } \multicolumn{1}{c|}{} & MRR & MR & Hits@ 10 & Hits@ 1 & MRR & MR & Hits@ 10 & Hits@ 1 \\
\hline TTRANSE & $\mathbf{3 2 . 1}$ & 578 & 51.0 & 23.0 & 48.8 & 80 & 80.6 & 33.9 \\
\hline \hline TRANSE & 29.6 & 614 & 46.8 & 22.8 & 31.6 & $\mathbf{5 0}$ & 65.9 & 18.1 \\
DISTMULT & 27.5 & 578 & 43.8 & 21.5 & 31.6 & 77 & 66.1 & 18.1 \\
\hline TA-TRANSE & $\mathbf{3 2 . 1}$ & 564 & $\mathbf{5 1 . 2}$ & $\mathbf{2 3 . 1}$ & 48.4 & 79 & $\mathbf{8 0 . 7}$ & 32.9 \\
TA-DISTMULT & 29.1 & $\mathbf{5 5 1}$ & 47.6 & 21.6 & $\mathbf{7 0 . 0}$ & 198 & 78.5 & $\mathbf{6 5 . 2}$ \\
\hline
\end{tabular}

Table 3: Results (filtered setting) of the temporal knowledge graph completion experiments for the data sets YAGO15K and WIKIDATA. The best results are written bold.

\begin{tabular}{|l|cccc|cccc|}
\cline { 2 - 9 } \multicolumn{1}{c|}{} & \multicolumn{4}{c|}{ ICEWS 2014 } & \multicolumn{4}{c|}{ ICEWS 2005-15 } \\
\cline { 2 - 9 } \multicolumn{1}{c|}{} & MRR & MR & Hits @ 10 & Hits@ 1 & MRR & MR & Hits@ 10 & Hits@ 1 \\
\hline TTRANSE & 25.5 & 148 & 60.1 & 7.4 & 27.1 & 181 & 61.6 & 8.4 \\
\hline \hline TRANSE & 28.0 & $\mathbf{1 2 2}$ & 63.7 & 9.4 & 29.4 & 84 & 66.3 & 9.0 \\
DISTMULT & 43.9 & 189 & 67.2 & 32.3 & 45.6 & 90 & 69.1 & 33.7 \\
\hline TA-TRANSE & 27.5 & 128 & 62.5 & 9.5 & 29.9 & $\mathbf{7 9}$ & 66.8 & 9.6 \\
TA-DISTMULT & $\mathbf{4 7 . 7}$ & 276 & $\mathbf{6 8 . 6}$ & $\mathbf{3 6 . 3}$ & $\mathbf{4 7 . 4}$ & 98 & $\mathbf{7 2 . 8}$ & $\mathbf{3 4 . 6}$ \\
\hline
\end{tabular}

Table 4: Results (filtered setting) of the temporal knowledge graph completion experiments for the data sets ICEWS 2014 and ICEWS 2005-15. The best results are written bold.

ICEWS data sets, YAGO15K does contain temporal modifiers; namely, 'occursSince' and 'occursUntil'. Contrary to previous work (Leblay and Chekol, 2018), all facts maintain time information in the same level of granularity as one can find in the original dumps these data sets come from.

We also experimented with the temporal facts from the WIKIDATA data set ${ }^{4}$ extracted in (Leblay and Chekol, 2018). Only information regarding the year is available for these facts, since the authors discarded information of finer granularity. All facts are framed in a time interval (i.e. they contain the temporal modifiers 'occursSince' and 'occursUntil'). Facts annotated with a single point-in-time are associated with that time-point as start and end time. Due to the large number of entities of this data set, which hinders the computation of standard KG completion metrics, we selected a subset of the most frequent entities and

\footnotetext{
${ }^{4}$ http://staff.aist.go.jp/julien.leblay/datasets
}

kept all facts where both the subject and object are part of this subset of entities. This set of filtered facts was split into training, validation and test in the same proportion as before.

Table 2 lists some statistics of the temporal KGs. All four data sets, with their corresponding training, validation, and test splits are available at https://github.com/nle-ml/mmkb.

\subsection{General Set-up}

We evaluate various methods by their ability to answer completion queries where i) all the arguments of a fact are known except the subject entity, and ii) all the arguments of a fact are known except the object entity. For the former we replace the subject by each of the KBs entities $\mathcal{E}$ in turn, sort the triples based on the scores returned by the different methods, and computed the rank of the correct entity. We repeated the same process for the second completion task and average the results. 
[playsFor, since, temporal_tokens(date)]

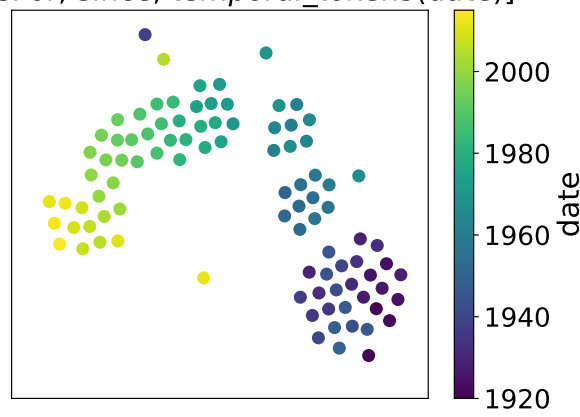

Figure 2: T-SNE visualization of the embeddings learned for the predicate sequence $p_{\text {seq }}=$ [playsFor, occursSince, date], where date corresponds to the date token sequence.

This is standard procedure in the KG completion literature. We also report the filtered setting as described in (Bordes et al., 2013). The mean of all computed ranks is the Mean Rank (lower is better) and the fraction of correct entities ranked in the top $n$ is called hits@n (higher is better). We also compute the Mean Reciprocal Rank (higher is better) which is less susceptible to outliers.

Recent work (Leblay and Chekol, 2018) evaluates different approaches for performing link prediction in temporal KGs. The approach that learns independent representations for each timestamp and use these representations as translation vectors, similarly to (Bordes et al., 2013), leads to the best results. This approach is called VECTORBASED TTRANSE, though for the shake of simplicity in the paper we refer to it as TTRANSE. We compare our approaches TA-TRANSE and TADISTMULT against TTRANSE, and the standard embedding methods TRANSE and DISTMULT. For all approaches, we used ADAM (Kingma and $\mathrm{Ba}, 2014$ ) for parameter learning in a mini-batch setting with a learning rate of 0.001 , the categorical cross-entropy (Kadlec et al., 2017) as loss function and the number of epochs was set to 500 . We validated every 20 epochs and stopped learning whenever the MRR values on the validation set decreased. The batch size was set to 512 and the number of negative samples to 500 for all experiments. The embedding size is $\mathrm{d}=100$. We apply dropout (Srivastava et al., 2014) for all embeddings. We validated the dropout from the values $\{0,0.4\}$ for all experiments. For TA-TRANSE and TA-DISTMULT, the activation gate $\sigma_{g}$ is the sigmoid function; $\sigma_{c}$ and $\sigma_{h}$ were chosen to be linear activation functions.

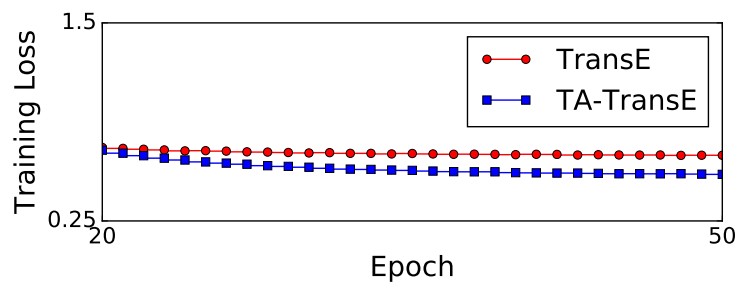

Figure 3: Training loss in YAGO15K. TATRANSE's ability to learn from time information leads to a lower loss.

\subsection{Results}

Table 3 and 4 list the results for the KG completion tasks. TA-TRANSE and TA-DISTMULT systematically improve TRANSE and DISTMULT in MRR, hits@10 and hits@1 in almost all cases. Mean rank is a metric that is very susceptible to outliers and hence these improvements are not consistent. TTRANSE learns independent representations for each timestamp contained in the training set. At test time, timestamps unseen during training are represented by null vectors. This explains that TTRANSE is only competitive in YAGO15K, wherein the number of distinct timestamps is very small (see \#Distinct TS in Table 2) and thus enough training examples exist to learn robust timestamp embeddings. TTRANSE's performance is similar to that of TA-TRANSE, our time-aware version of TRANSE, in WIKIDATA. Similarly, TTRANSE can learn robust timestamp representations because of the small number of distinct timestamps of this data set.

Figure 3 shows a comparison of the training loss of TRANSE and TA-TRANSE for YAGO15K. Under the same set-up, TA-TRANSE's ability to learn from time information leads to a training loss lower than that of TRANSE.

Figure 2 shows a t-SNE (Maaten and Hinton, 2008) visualization of the embeddings learned for the predicate sequence $p_{\text {seq }}=$ [playsFor, occursSince, date], where date corresponds to the date token sequence. This illustrates that the learned relation type embeddings carry temporal information.

\section{Conclusions}

We propose a digit-level LSTM to learn representations for time-augmented KG facts that can be used in conjunction with existing scoring functions for link prediction. Experiments in four temporal knowledge graphs show the effectiveness of the approach. 


\section{References}

J. van Benthem. 1995. Handbook of logic in artificial intelligence and logic programming (vol. 4). chapter Temporal Logic, pages 241-350. Oxford University Press, Inc., New York, NY, USA.

Antoine Bordes, Nicolas Usunier, Alberto GarciaDuran, Jason Weston, and Oksana Yakhnenko. 2013. Translating embeddings for modeling multirelational data. In Advances in neural information processing systems, pages 2787-2795.

Melisachew Wudage Chekol, Giuseppe Pirrò, Joerg Schoenfisch, and Heiner Stuckenschmidt. 2017. Marrying uncertainty and time in knowledge graphs. In $A A A I$, pages 88-94.

Melisachew Wudage Chekol and Heiner Stuckenschmidt. 2018. Rule Based Temporal Inference. In Technical Communications of the 33rd International Conference on Logic Programming (ICLP 2017), volume 58, pages 4:1-4:14, Dagstuhl, Germany. Schloss Dagstuhl-Leibniz-Zentrum fuer Informatik.

Maximilian Dylla, Iris Miliaraki, and Martin Theobald. 2013. A temporal-probabilistic database model for information extraction. Proc. VLDB Endow., 6(14):1810-1821.

C. Esteban, V. Tresp, Y. Yang, S. Baier, and D. Krompa. 2016. Predicting the co-evolution of event and knowledge graphs. In 2016 19th International Conference on Information Fusion (FUSION), pages 98-105.

Alberto Garcia-Duran and Mathias Niepert. 2017. Kblrn: End-to-end learning of knowledge base representations with latent, relational, and numerical features. arXiv preprint arXiv:1709.04676.

Kelvin Guu, John Miller, and Percy Liang. 2015. Traversing knowledge graphs in vector space. arXiv preprint arXiv:1506.01094.

Johannes Hoffart, Fabian M Suchanek, Klaus Berberich, and Gerhard Weikum. 2013. Yago2: A spatially and temporally enhanced knowledge base from wikipedia. Artificial Intelligence, 194:28-61.

Tingsong Jiang, Tian Yu Liu, Tao Ge, Lei Sha, Baobao Chang, Sujian Li, and Zhifang Sui. 2016. Towards time-aware knowledge graph completion. In $C O L$ ING.

Rudolf Kadlec, Ondrej Bajgar, and Jan Kleindienst. 2017. Knowledge base completion: Baselines strike back. arXiv preprint arXiv:1705.10744.

Yoon Kim, Yacine Jernite, David Sontag, and Alexander M Rush. 2016. Character-aware neural language models. In $A A A I$, pages 2741-2749.

Diederik P Kingma and Jimmy Ba. 2014. Adam: A method for stochastic optimization. arXiv preprint arXiv:1412.6980.
Julien Leblay and Melisachew Wudage Chekol. 2018. Deriving validity time in knowledge graph. In Companion Proceedings of the The Web Conference 2018, WWW '18. International World Wide Web Conferences Steering Committee.

Laurens van der Maaten and Geoffrey Hinton. 2008. Visualizing data using t-sne. Journal of machine learning research, 9(Nov):2579-2605.

Maximilian Nickel, Kevin Murphy, Volker Tresp, and Evgeniy Gabrilovich. 2016. A review of relational machine learning for knowledge graphs. Proceedings of the IEEE, 104(1):11-33.

Nitish Srivastava, Geoffrey Hinton, Alex Krizhevsky, Ilya Sutskever, and Ruslan Salakhutdinov. 2014. Dropout: A simple way to prevent neural networks from overfitting. The Journal of Machine Learning Research, 15(1):1929-1958.

Rakshit Trivedi, Hanjun Dai, Yichen Wang, and Le Song. 2017. Know-evolve: Deep temporal reasoning for dynamic knowledge graphs. In Proceedings of the 34th International Conference on Machine Learning, volume 70 of Proceedings of Machine Learning Research, pages 3462-3471, International Convention Centre, Sydney, Australia. PMLR.

Bishan Yang, Wen-tau Yih, Xiaodong He, Jianfeng Gao, and Li Deng. 2014. Learning multi-relational semantics using neural-embedding models. arXiv preprint arXiv:1411.4072.

Xiang Zhang, Junbo Zhao, and Yann LeCun. 2015. Character-level convolutional networks for text classification. In Advances in neural information processing systems, pages 649-657. 